\title{
Risk Factors for Suicide in Taiwanese College Students
}

\author{
Susan Shur-Fen Gau, MD, PhD; Ying-Yeh Chen, MD, ScD; Fang-Ju Tsai, MD; \\ Ming-Been Lee, MD; Yen-Nan Chiu, MD; Wei-Tsuen Soong, MD, FRCP(C); \\ Hai-Gwo Hwu, MD
}

\begin{abstract}
Objective: The authors investigated the personality characteristics, psychopathology, parenting style, and family function among Taiwanese college students with high, moderate, and low suicidal risks. Participants: The sample included 2,919 first-year college students (1,414 men, 1,505 women) from a university in Taipei, Taiwan. Methods: A self-administered questionnaire assessed domains covering demographics, personality, psychopathology, frequency of substance use, parenting style, family functioning, and suicidal behaviors. The authors used mixed models for data analysis. Results: The authors observed a positive linear trend between increased suicidal tendency and levels of neuroticism, harm avoidance, novelty seeking, psychopathology, and parenting styles of low affection, overprotection, and authoritarian controlling. Use of tobacco and alcohol and impaired family adaptation and cohesion were associated with high and moderate suicidal risks. Conclusions: Personality, psychopathology, substance use, and familial factors are important correlates of suicidal risks among college students in Taiwan. Optimal suicide prevention strategies in the college setting should incorporate the multiple facets of suicidal risks.
\end{abstract}

Keywords: college students, parenting style, personality, psychopathology, suicidal risk

uicide is one of the leading causes of death among young adults worldwide. ${ }^{1,2}$ Many parts of the world experienced a marked increase in suicide rate in the 15-24 age group after 1950. ${ }^{3}$ In Asian countries, however, youth suicide rates have not experienced such an acute increase as have been observed in the Western world. ${ }^{4}$ In

Drs Gau, Lee, Chiu, Soong, and $\mathrm{Hwu}$ are with the Department of Psychiatry at National Taiwan University Hospital, Taipei. Drs Gau, Lee, Soong, and Hwu are also with the Department of Psychiatry, College of Medicine at National Taiwan University. Dr Chen is with Taipei City Hospital, Songde Branch, Taipei, Taiwan, and the College of Medicine at National Yang Ming University, Taiwan. Dr Tsai is with the Department of Psychiatry at En Chu Kong Hospital, Taipei, Taiwan.

Copyright () 2008 Heldref Publications the recent period, a small, gradual increase in youth suicide rates has been observed in some Asian countries. In Taiwan, for example, youth suicide rates were 4.40 per 100,000 population in 1994 and increased to 6.21 per 100,000 population in 2004. During this period, suicide death has jumped from the third leading cause of death (accounting for $4.5 \%$ of deaths) to the second leading cause of death (accounting for $10.5 \%$ of deaths) among the 15-24 age group. ${ }^{5}$ Investigating antecedents and correlates of suicidal behaviors is thus important in tackling this disturbing trend of youth suicide.

Suicide is not an isolated event; it is a sequence of process starting from death wishes, suicidal ideation, suicidal contemplation, suicide attempt, and, finally, suicide completion. ${ }^{6,7}$ Studies have shown that different types of suicidal cognitions (eg, death wishes, suicidal ideations, suicide plan) and behaviors (eg, suicide attempt, committed suicide) share common determinant factors, such as personality characteristics, psychopathology, parenting styles, family function, and substance abuse. ${ }^{7-9}$ Findings from one longitudinal study demonstrated that individuals characterized by high levels of novelty seeking and neurotic traits were more vulnerable to both suicidal ideation and suicide attempt. ${ }^{10}$ Psychopathology—such as anxiety, depression, psychosis, and substance use disorders-has consistently been reported to be associated with a variety of suicidal expressions. ${ }^{11,12}$ Inappropriate parenting styles-either overprotection or inadequate care-and poor family function significantly increase the risks of suicidal ideation and suicide attempts. ${ }^{13,14}$

Although previous researchers have documented the association between the previously mentioned risk factors and a variety of suicidal intent and behaviors, these factors have not been well investigated in the Chinese population. Furthermore, the linear relationship between suicidal risk factors and suicidal intent has not been well established. In other words, 
there is little information as to whether the intensity of psychosocial risks for suicide corresponds to an increase in the severity of suicidal intent. In view of this, we conducted a cross-sectional school-based survey at a national university in Taipei. Our objectives were to identify personality characteristics, psychopathology, parenting styles, and family functioning among college students who were at high, moderate, and low risk of suicide. We hypothesized that college students at different levels of risk would demonstrate differential psychological, behavioral, and familial patterns.

\section{METHODS}

\section{Participants and Procedure}

The university's institutional review board approved this study prior to implementation. In July 2002, we mailed a letter describing the purposes and procedures of the study to incoming freshmen. The letter stated that participation in the survey was voluntary and assured confidentiality. Among 3,688 first-year students, 2,919 (48.5\% male, $51.5 \%$ female, mean age $\pm S D, 19.4 \pm 3.0$ ) consented to and completed the self-administered questionnaire survey in the first week of the fall semester in conjunction with a routine physical examination (participation rate $=79.2 \%$ ). We have no information about the proportion of eligible students who received the letter.

\section{Student Counseling Center}

At the time of the study, the student counseling center staff comprised 1 director (whose educational background is in psychiatry and public health), 9 full-time board-certificated clinical or counseling psychologists, 1 full-time social worker, 10 part-time clinical psychologists, and 4 part-time psychiatrists. The facility, a training center for counseling psychology, provides primary, secondary, and tertiary school mental health prevention in the format of psychological assessments, workshops, student camping, educational symposia, individual counseling and psychotherapy, family therapy, group therapy, regular school survey, follow-up with high-risk students, and establishment of a referral system with community psychological service and hospital psychiatric service.

\section{Outcome Measure: Low, Moderate, and High Suicide Risk}

We divided participants into 3 groups according to their current (past 6 months) and past suicide-related behaviors: (1) high suicidal risk, which comprised students who reported current suicidal ideation, wishes, or plans or had ever attempted suicide ( $n=250)$; (2) moderate suicidal risk, comprising students who had suicidal ideation, wishes, or plans in the past $(n=536)$; and (3) low suicidal risk, comprising students who never had any suicidal thoughts or plans $(n=2,133)$.

After survey, they received 1 of 3 letters designed for each of the groups, with an explanation of the survey results and including information about the services provided by the university health center. In addition, school counselors called students who scored as being at high suicidal risk immediately after they received the results and provided individual counseling, psychotherapy, group therapy, or workshops. Counselors invited moderate-risk students to visit the health center whenever they needed counseling. Among the 250 high-risk students, 26 (10.4\%) provided no contact information. At 3-month follow-up, 196 (78.4\%) of the high-risk students improved without any suicidal ideation, $23(9.2 \%)$ had no obvious improvement and needed continued individual counseling, and 5 (2.0\%) had an exacerbated condition and received combined counseling and psychiatric treatment.

\section{Measures for Independent Variables}

\section{Personality Characteristics}

\section{Maudsley Personality Inventory}

The Maudsley Personality Inventory (MPI), a 30-item self-administered scale, measures 3 personality traits-neuroticism, extraversion, and social desirability-with a yes or no response. The Chinese MPI has proven to be a reliable and valid instrument for use in both community and medical settings in Taiwan. ${ }^{15}$ In our sample, internal consistency was high for neuroticism $(\alpha=.79)$ and extraversion $(\alpha=.76)$. We did not include the social desirability subscale in the analysis because of its low internal consistency $(\alpha=.32)$.

\section{Chinese Version of the Tridimensional Personality Ques- tionnaire}

The Tridimensional Personality Questionnaire (TPQ) contains 100 items measuring 3 personality dimensions: novelty seeking (NS), harm avoidance (HA), and reward dependence (RD). Researchers ${ }^{16,17}$ have examined the Chinese TPQ's reliability and validity among both adult and adolescent samples. In our study sample, the Cronbach alpha coefficients for internal consistency reliability were .72 for NS, .87 for HA, and .63 for RD.

\section{Psychopathology and Substance Use}

\section{Psychopathology: Brief Symptom Rating Scale}

The 50-item self-report Brief Symptom Rating Scale (BSRS) is a modification of the Derogatis Symptom Check List 90-Revised (SCL-90-R), ${ }^{18}$ covering 9 dimensions of psychopathology: somatization, obsessive-compulsive, interpersonal sensitivity, depression, anxiety, hostility, phobic-anxiety, paranoid ideation, and additional symptoms including vegetative signs and suicidal ideation. Respondents rate each item on a 4-point Likert scale from 0 (not at all) to 4 (extremely). The General Symptom Index (GSI) is a mean score of all BSRS categories. The reliability and validity of BSRS has been demonstrated elsewhere. ${ }^{19}$ In this study, internal consistency $(\alpha)$ of the 10 BSRS subscales ranged from .68 to .96 .

\section{Substance Use}

Questions measuring substance abuse included regular use (at least once per week) of nicotine, wine or beer, 
liquor, betel nut, glue, sedatives, and amphetamines. The kappa statistics for test-retest reliability at a 2-week interval among 115 respondents ranged from 0.67 for liquor to 1.00 for betel nuts, glue, sedatives, or amphetamines.

\section{Parenting and Family Functioning}

\section{Chinese Version of the Parental Bonding Instrument}

The Parental Bonding Instrument (PBI) is a 25-item instrument (rated on a 4-point Likert scale from 1 [very likely] to 4 [very unlikely]) measuring parenting styles during the child's first 16 years, using 2 principal dimensions-care (12 items) and protection (13 items). High scores on the care subscale reflect affection and warmth; low scores indicate rejection or indifference. The protection scale can be further divided into authoritarianism (6 items), reflecting the degree of parental authoritarian control over the child's behavior, and overprotectiveness (7 items), reflecting overprotective parenting and denial of the child's psychological autonomy. ${ }^{20}$ The reliability and validity of the Chinese version of the PBI (PBI-C) has been demonstrated elsewhere. ${ }^{21}$ In this study, the Cronbach alpha for internal consistency and reliability of the 3 PBI-C subscales ranged from .82 to .90 for reports on fathering and from .81 to .90 for reports on mothering.

\section{Chinese Version of the Family Adaptability and Cohesion Evaluation Scale}

Olson $^{22}$ developed the Family Adaptability and Cohesion Evaluation Scale (FACESIII), a 40-item self-reporting scale, to assess family systems with respect to the levels of current and ideal adaptability and cohesion. Respondents rate each item on a 5-point Likert scale-1 (almost never), 2 (once in a while), 3 (sometimes), 4 (frequently), and 5 (almost always). In addition to current and ideal conditions, we computed differences in family cohesion and adaptability. A high score indicates better family functioning. In this study, internal consistency was high for the 4 subscales $(\alpha=.77-.88)$.

\section{Covariates}

We included in the analysis demographic factors such as age, sex, and residential areas to control for their potential confounding effects.

\section{Statistical Analysis}

We conducted statistical analysis using SAS 9.1 (SAS Institute Inc, Cary, NC). We used linear and nonlinear mixed models to control for any clustering effects derived from a college-based sample. We used nonlinear mixed models for logistic regression to examine the association between suicidal risk and substance use to then generate the odds ratios (ORs) and 95\% confidence intervals (CIs). We used a goodness of fit test with chi-square distribution to test for a linear trend among the 3 levels of suicidal risk. We used the Scheffe method in the mixed model to adjust for multiple comparisons among the 3 suicidal risk groups. The preselected alpha value was at the $p<.05$ level. (The statistical details of this study are available on request from the lead author [S.S.F. Gau].)

\section{RESULTS}

Of the 2,919 participants, $30(1.0 \%)$ and $759(26.0 \%)$ students currently had (within the past 6 months) and ever had suicidal ideations, respectively. The mean age $(S D)$ of onset of suicidal ideation was 13.7 years (3.6). Twenty-three students $(0.8 \%)$ currently wished they were dead, and 642 students $(22.0 \%)$ had ever wished they were dead. The mean age $(S D)$ of onset of suicidal wish was 13.8 years (3.6). Six students currently had plans of killing themselves, and 220 students (7.5\%) had ever planned to kill themselves. The mean age $(S D)$ at which they had had their first suicidal plans was 14.2 years (4.2). Although none reported attempting suicide within the past 6 months, 34 students had ever attempted to kill themselves. The mean age $(S D)$ of suicidal attempts was 14.2 years (3.4).

Figure 1 presents the mean scores of neuroticism, extraversion, novelty seeking, harm avoidance, and reward dependence of the 3 suicidal groups. We found a positive linear relationship between the hierarchy of suicidal risks and the tendency of neuroticism, novelty seeking, and harm avoidance. Extraversion tendency was protective against suicide: increased levels of extraversion were related to a graded decrease in suicidal risks. We observed no differences in reward dependence among the 3 groups.

Figure 2 shows the mean scores for each symptom dimension of the BSRS by suicidal risk group. In making this comparison, we observed a linear relationship between increased risk for suicide and increased severity of psychiatric symptoms.

Figure 3 presents the rates and ORs for regular substance use, by risk group. Compared with the low-risk suicidal group, the high- and moderate-risk groups had significantly higher odds of using tobacco and drinking alcohol. We found no difference between the high- and moderate-risk groups in terms of smoking and drinking alcohol. The number and rates for use of betel nuts $(42,1.44 \%)$, illicit sedatives $(11,0.38 \%)$, glue $(11,0.38 \%)$, and amphetamines $(4,0.14 \%)$ were too low to allow detection of differences among the 3 groups.

Figures 4 and 5 present the scores of the subscales of the PBI for parenting styles (Figure 4) and of the FACESIII for family functioning (Figure 5). Participants at high risk of attempting suicide reported their mothers and fathers to be less affectionate and caring as well as more overprotective and authoritarian controlling than did the moderate-risk group, followed by the low-risk group. The relationships were arranged in a gradient response pattern. In terms of family adaptability and cohesion and the differences between the current and ideal family functioning - regardless of whether they were the current or ideal situation-we found that high- and moderate-risk participants reported poorer family adaptability and cohesion than did low-risk participants. The reported differences between ideal and current family functioning were greater among high- or moderate-risk participants. 


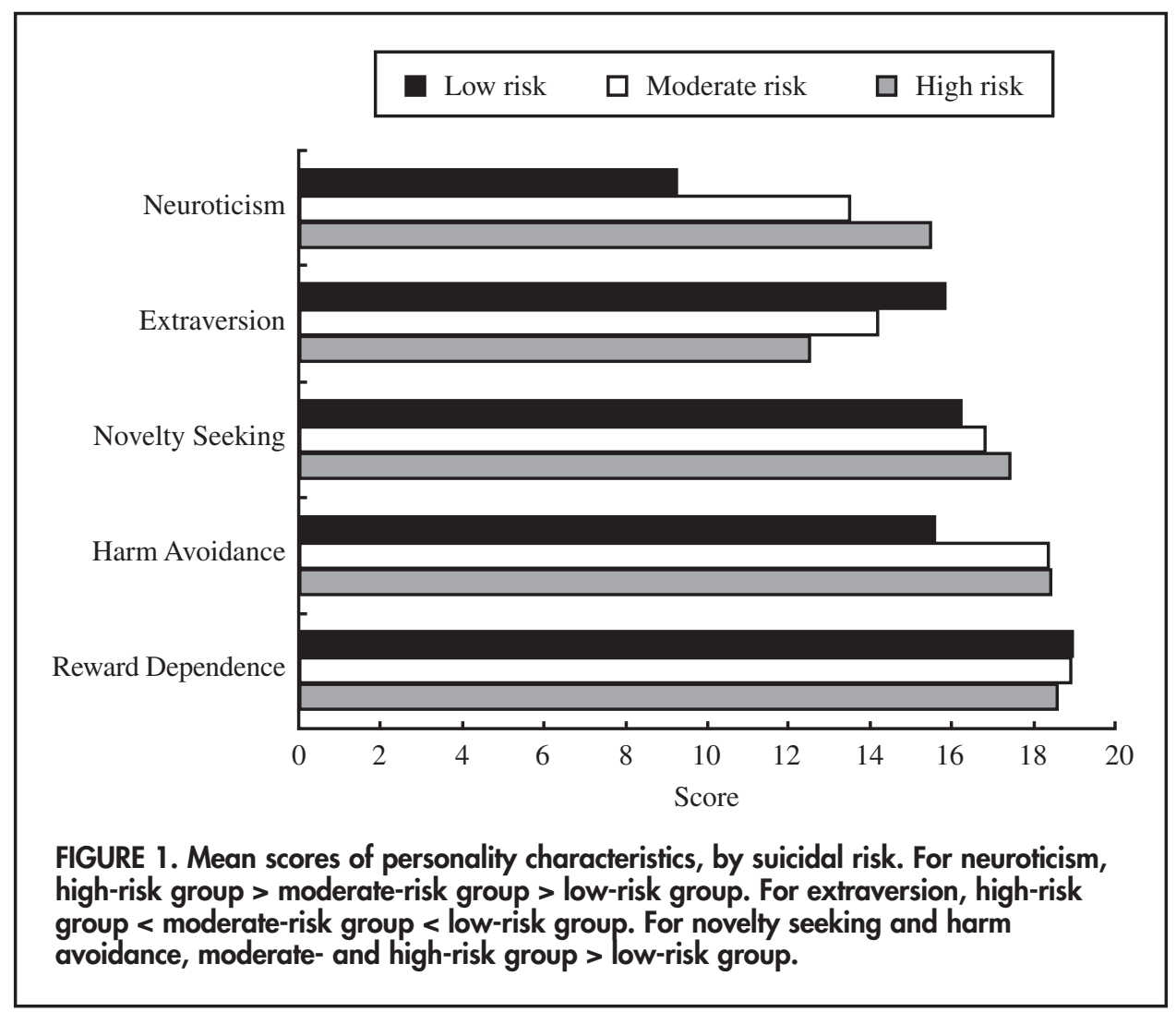

\section{COMMENT}

We found that personality problems, psychopathology, and inappropriate parenting style had a consistent gradient effect on the intensity of suicidal risk. Because a gradient response relationship is an important indicator for causality, ${ }^{23}$ our study thus verifies the role of these psychosocial factors in predicting suicidal behavior among college students in Taiwan. Although the relationships between substance abuse, family functioning, and suicide risk were not arranged in a gradient pattern, the low-risk group reported the least amount of substance use and healthiest family functioning. Research on suicidal intensity and correlates for suicidal behaviors among the Chinese population has been sparse; therefore, the current findings provide valuable information for international comparisons.

Consistent with previous Western research, ${ }^{10,24}$ our results demonstrate that neuroticism, introversion, novelty seeking, and harm avoidance are attributes associated with suicide risk. Individuals with neurotic tendency or low levels of extraversion are less likely to seek help, tend to interpret things negatively, and are therefore predisposed to hopelessness, depression, and suicide. ${ }^{24}$ Novelty seeking is associated with impulsivity and substance abuse; therefore, it may increase the risk for suicide. ${ }^{25}$ Harm avoidance is an important indicator for anxiety proneness, which is one of the documented correlates of suicidal tendency. ${ }^{24}$

Regarding psychopathology, the literature reveals a consistent pattern of association between mental disorders and suicide attempts ${ }^{26}$ and completions. ${ }^{27}$ Our results replicate previous findings that psychiatric symptoms-including somatization, obsession, interpersonal sensitivity, depression, anxiety, hostility, phobia, paranoia, psychoticism, and other symptoms (eg, vegetative signs) — correlate positively with suicidal risk in a gradient pattern. ${ }^{28,29}$ Our findings also support a relationship between substance abuse (including cigarette smoking ${ }^{30}$ and drinking ${ }^{31}$ ) and the suicide risks of college youths.

To our knowledge, our finding of the effect that individual personality traits and psychiatric symptoms have on suicide risk in a gradient response pattern has not been reported in previous studies, possibly because a threshold effect is often implicitly assumed. That is to say, existing literature provides information only on whether a certain factor increases risk for suicide but does not explore whether the increased magnitude of that factor increases the intensity level of suicidal risks. Because risk factors' gradient patterns imply that identification of the high-risk group is artificial, our finding therefore suggests a more generalized approach to promote overall mental health among college students, rather than merely focusing on the at-risk population. One possible intervention is designing appropriate curriculum to enhance students' coping skills and self-esteem (eg, assertive training, self-efficacy strengthening), thus helping students become mature enough to cope with the difficulties they encounter. ${ }^{32}$ Instead of focusing on certain problematic students, this generalized approach aims to improve students' mental health as a whole, and, thus, overall suicidal risk will decrease. As described in the Methods section, we 


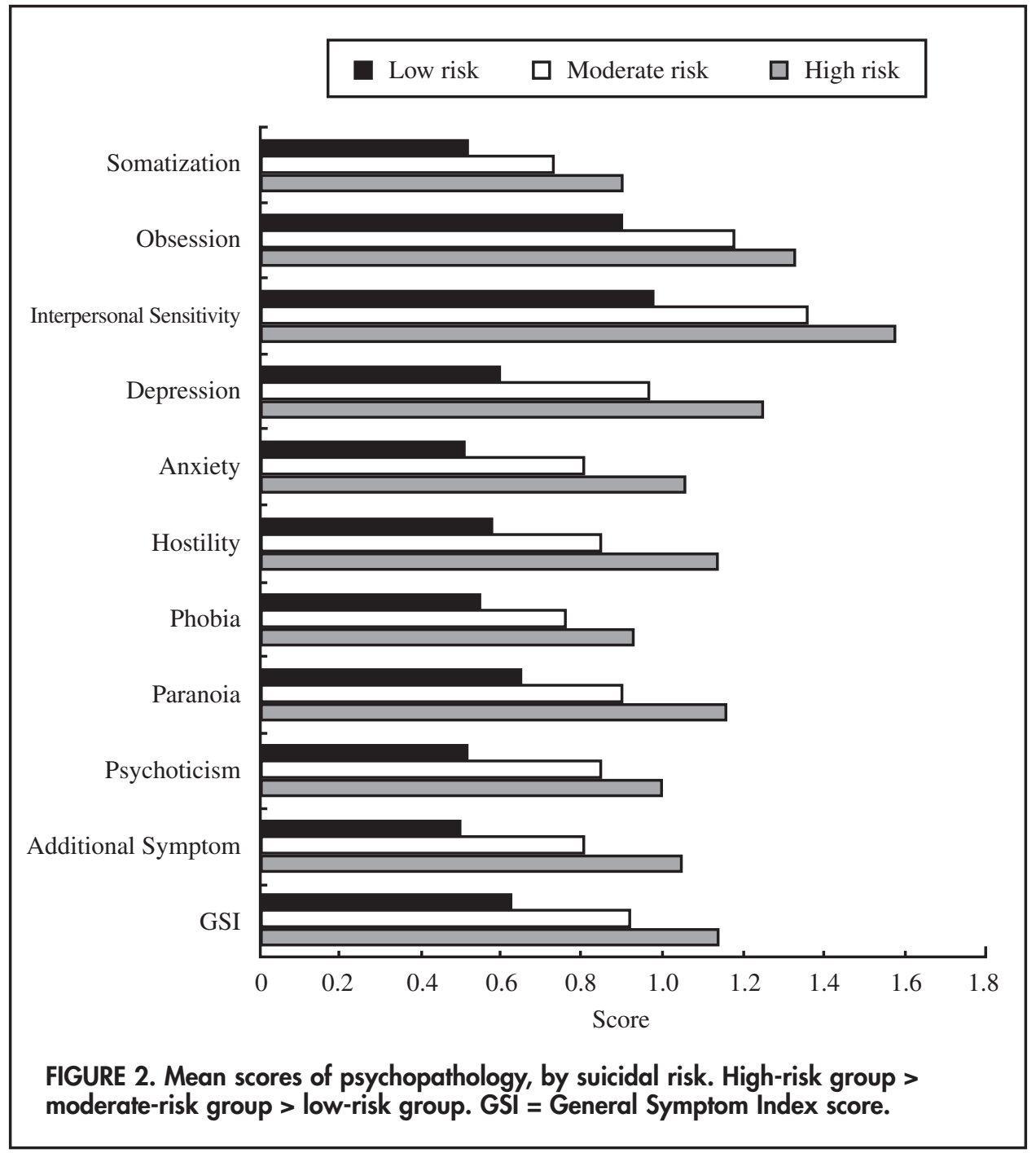

have done extensive work to improve school mental health approaches, promoting both a general and an individual approach for high-risk students.

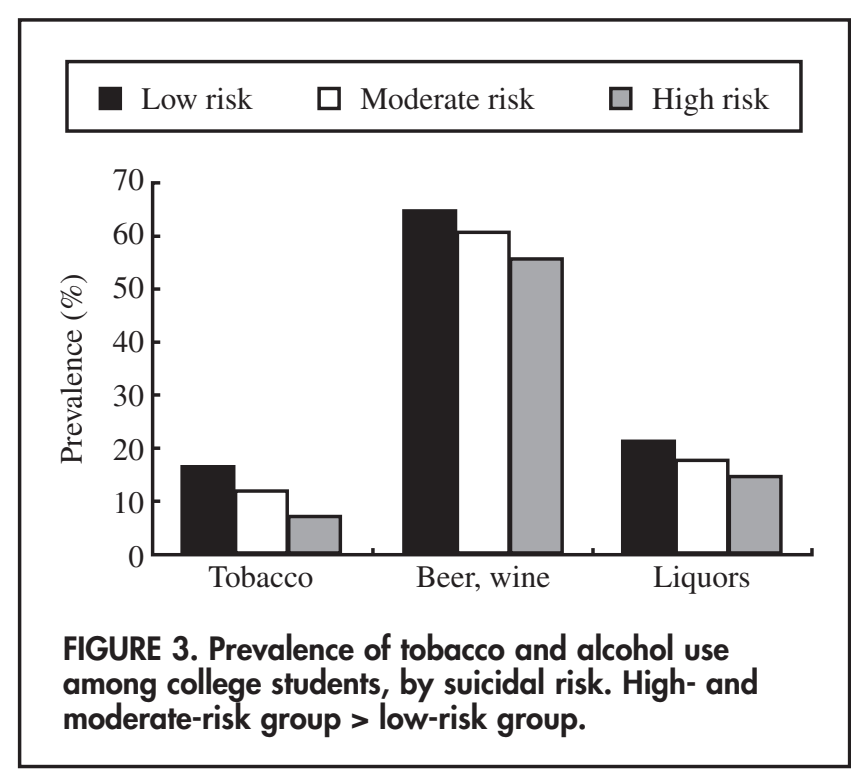

VOL 57, SEPTEMBER/OCTOBER 2008
Turning to familial factors, consistent with previous research, we found that when students reported parents showing adequate care and affection, those students' suicidal risk was ameliorated; when parents were perceived as overprotective or authoritarian controlling, suicidal risks increased in a graded fashion. ${ }^{33}$ Chinese parenting style is often described as "authoritarian," "controlling," or "restrictive."32(p1111) According to cross-cultural studies, authoritarian parenting style is negatively associated with academic achievement among European American students but positively related to high academic achievement among Chinese students. ${ }^{34,35}$ Although some argue that the authoritarian parenting style carries a negative meaning in the Western context, this may not be the case in the Asian society. ${ }^{34}$ For example, in Chinese culture, "authoritarian" may mean "child training" or "intensive parental care" and is therefore not necessarily negative. ${ }^{34}$ In our analysis, however, we found that perceived overprotectiveness and authoritarian controlling increased college students' suicide risk, even in the context of Chinese culture. Thus, our findings regarding the influence of inappropriate parenting style on suicidal risk should be cross-culturally valid. Future 


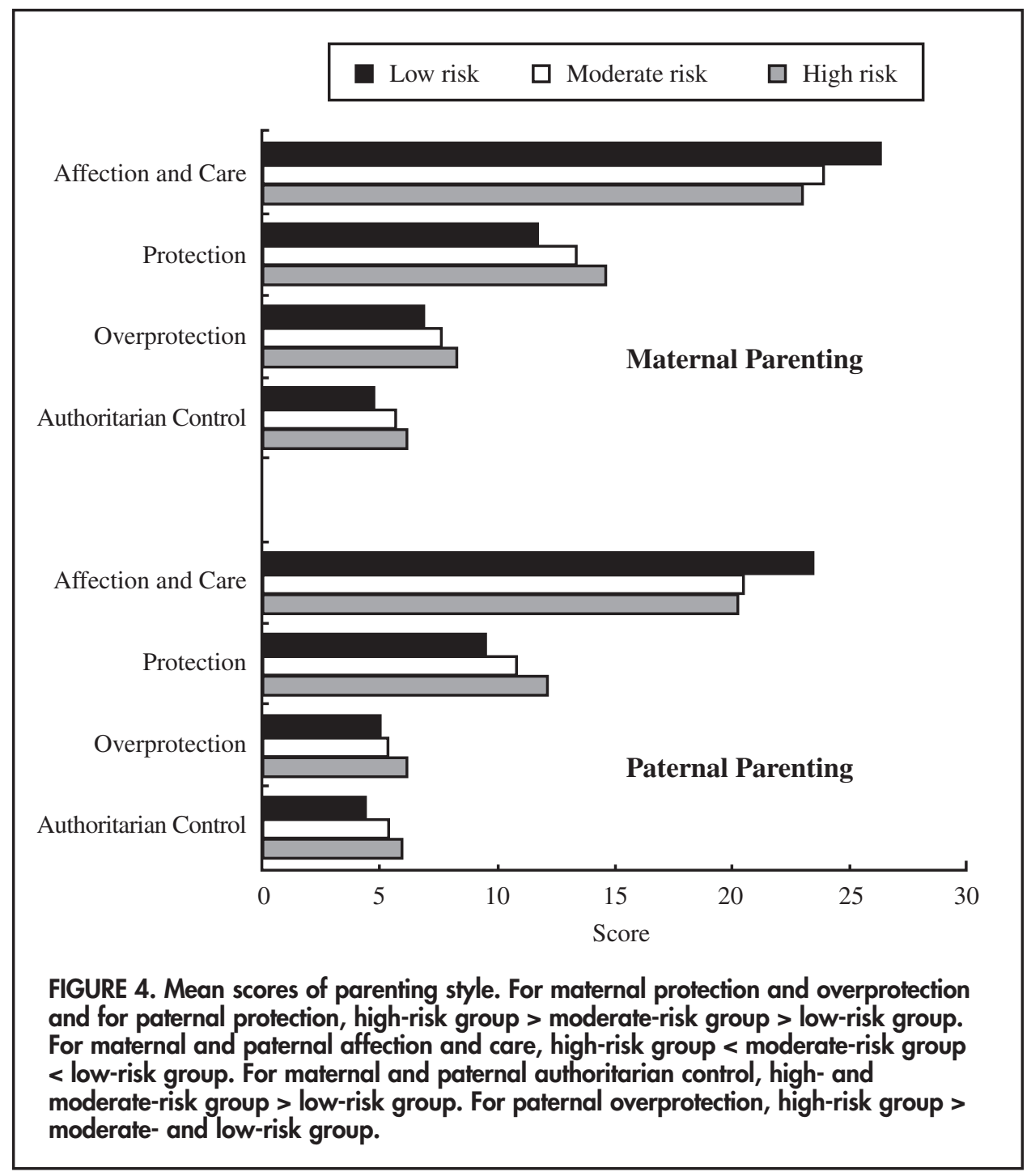

researchers should examine the influence of parenting style on children's mental health outcomes not only across different cultures, but also across different subgroups in the same culture.

Like mothers in Western culture, Taiwanese mothers have more child-care responsibilities than do fathers. Influenced by the Confucian practice, Chinese families emphasize patriarchy and children's academic performance. To ensure children's well-being, fathers often set the rules of conduct in the household and mothers make sure these rules are followed. Therefore, mothers are responsible for children's performance and achievements. However, our study reveals that not only does maternal parenting matter, but also that paternal parenting determines children's suicide risk. However, compared with fathers, mothers were generally perceived as more caring and scored higher on the overprotection and authoritarian control subscale in this study, indicating that the perceived emotional tone is stronger in the mother-child relationship than in the father-child relationship.
Regarding perceived family support, our findings added some evidence that family cohesion is protective for suicidal behaviors ${ }^{36}$; as previously found, family dysfunction and poor family support accounted for suicidal risks among college youths. ${ }^{13,37}$ The influence of family dysfunction on college students' suicidal behaviors may work through other variables such as depression, which has been reported to be associated with perceived family dysfunction. ${ }^{37}$

The strength of our study lies in its provision of valuable information on suicide risk in a rarely studied population. Furthermore, the analysis covers a comprehensive list of suicidal risk factors, allowing us to more fully understand the facets of suicidal behaviors. Second, to our knowledge, we are the first to explore the gradient-response relationship of psychosocial factors and suicide risk. Our approach provides new insight in designing suicide intervention programs. Third, a wealth of reliable and valid self-reported measures provided us with a holistic and comprehensive picture of suicide risk correlates in this young adult population. Last, our participants comprised a relatively high- 


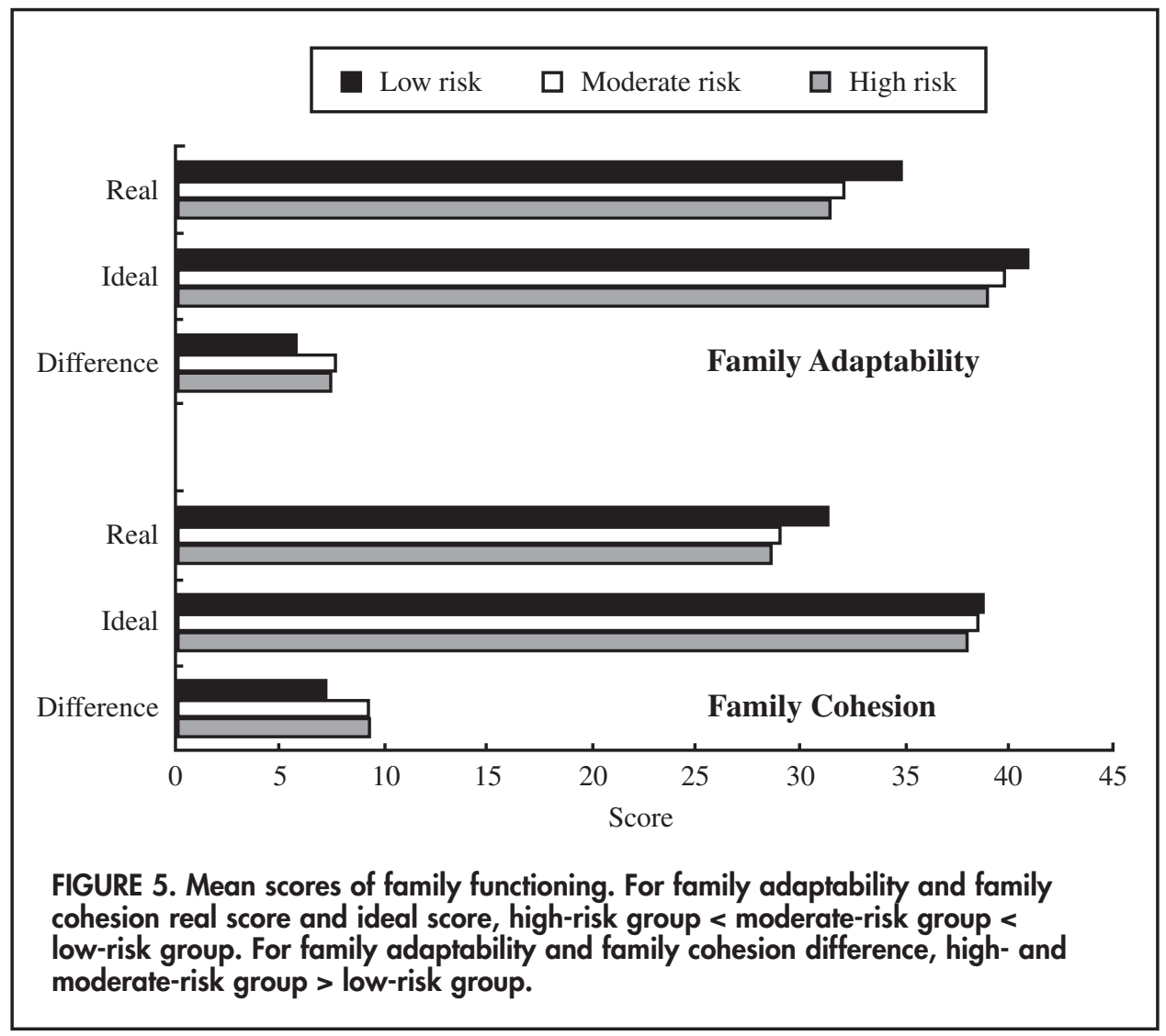

functioning group; the research is thus strengthened by its high response rate and excellent response quality.

Despite its strength, a number of limitations should be addressed in the interpretation of these results. First, the cross-sectional design limits our ability to make causal inference; causal effect of exposures and outcomes cannot be addressed-only suggested. Further research adopting a controlled prospective design is required to adequately address questions of causality and temporal sequencing. Second, findings from this study may not be generalized to other Taiwanese residents beyond those enrolled in this university. Last, all results were based on self-reported questionnaires rather than face-to-face interviews; therefore, discrete diagnoses were not available. However, self-report surveys are commonly used in school surveys in examining students' mental health and are generally considered to be valid. ${ }^{38}$

In conclusion, personal risk factors of suicide, including personality and psychopathology, were arranged in a graded fashion: in other words, increased severity of the problem was associated with increased suicide risk. Despite the fact that we conducted our study in Taiwan, our findings are consistent with those in Western countries; therefore, the findings and implications from this study can be applied cross-culturally. The implications of this study are that optimal suicide prevention strategies for college youths should consider their overall mental well-being, rather than focusing on just the at-risk individuals. Regarding family factors, both parenting style and family function are potent predictors of suicide risks among this population, suggesting the need to include family as a unit of intervention in designing suicide prevention programs.

\section{ACKNOWLEDGMENT}

This study was supported by funds from a National Taiwan University Hospital Award, NTUH 92-S07, and from the Office of Student Affairs, National Taiwan University.

\section{NOTE}

For comments and further information, address correspondence to Dr Susan Shur-Fen Gau, Department of Psychiatry, National Taiwan University Hospital and College of Medicine, No. 7, Chung-Shan South Road, Taipei 10002, Taiwan (e-mail: gaushufe@ntu.edu.tw).

\section{REFERENCES}

1. Madge N. Youth suicide in an international context. Eur Child Adolesc Psychiatry. 1999;8:283-291.

2. Pelkonen M, Marttunen M. Child and adolescent suicide: epidemiology, risk factors, and approaches to prevention. Paediatric Drugs. 2003;5:243-265.

3. Cantor CH. Suicide in the western world. In: Hawton K, Heeringen KV, eds. The International Handbook of Suicide and Attempted Suicide. New York: John Wiley \& Sons; 2000:9-28.

4. Lin JJ, Lu TH. Suicide mortality trends by sex, age, and method in Taiwan, 1971-2005. BMC Public Health. 2008;8:6.

5. Department of Health. Executive Yuan, Taiwan ROC. http:// www.doh.gov.tw/statistic. Accessed January 10, 2006.

6. Renberg ES. Self-reported life weariness, death wishes, suicidal ideation, suicidal plans and suicide attempts in general population surveys in the north of Sweden 1986 and 1996. Soc Psychiatry Psychiatr Epidemiol. 2001;36:429-436. 
7. Perez WV. The relationship between seriously considering, planning, and attempting suicide in the Youth Risk Behavior Survey. Suicide Life Threat Behav. 2005;35:35-49.

8 Beautrais AL. Risk factors for suicide and attempted suicide among young people. Aust N Z J Psychiatry. 2000;34:420-436.

9. Kuo PH, Yang HJ, Soong WT, Chen WJ. Substance use among adolescents in Taiwan: associated personality traits, incompetence, and behavioral/emotional problems. Drug Alcohol Depend. 2002;67:27-39.

10. Fergusson DM, Beautrais AL, Horwood LJ. Vulnerability and resiliency to suicidal behaviours in young people. Psychol Med. 2003;33:61-73.

11. Shaffer D, Gould MS, Fisher P, et al. Psychiatric diagnosis in child and adolescent suicide. Arch Gen Psychiatry. 1996;53:339-348.

12. Rowan AB. Adolescent substance abuse and suicide. Depress Anxiety. 2001;14:186-191.

13. Garber J, Little S, Hilsman, R, Weaver KR. Family predictors of suicidal symptoms in young adolescents. J Adolesc. 1998; 21:445-457.

14. Beautrais AL. Risk factors for serious suicide attempts among young people: a case control study. In: Kosky RJ, Eshkevari HS, Goldney RD, Hassan R, eds. Suicide Prevention: The Global Context. New York: Plenum; 1998:167-181.

15. Liao SC, Lee MB, Lee YG, Weng T, Shih FY, Ma HM Association of psychological distress and psychosocial factors in rescue workers within two months of a major earthquake. $J$ Formos Med Assoc. 2002;101:169-176.

16. Chen WJ, Chen HM, Chen CC, Chen CC, Yu WY, Cheng AT. Cloninger's Tridimensional Personality Questionnaire: psychometric properties and construct validity in Taiwanese adults. Compr Psychiatry. 2002;43:158-166.

17. Kuo PH, Chih YC, Soong WT, Yang HJ, Chen WJ. Assessing personality features and their relations with behavioral problems in adolescents: Tridimensional Personality Questionnaire and Junior Eysenck Personality Questionnaire. Compr Psychiatry. 2004;45:20-28.

18. Derogatis LR. SCL-90 R Manual I: Scoring, Administration, and Procedure for the SCL-90-R. Baltimore, MD: Clinical Psychometric Research, 1977.

19. Lee MB, Liao SC, Lee YJ, et al. Development and verification of validity and reliability of a short screening instrument to identify psychiatric morbidity. J Formos Med Assoc. 2003;102:6871-6894.

20. Cox BJ, Enns MW, Clara IP. The Parental Bonding Instrument: confirmatory evidence for a three-factor model in a psychiatric clinical sample and in the National Comorbidity Survey. Soc Psychiatry Psychiatr Epidemiol. 2000;35:353-357.

21. Gau SS, Shen HY, Chow MC, Tang CS, Chiu YN, Gau CS. Determinants of adherence to methylphenidate and the impact of poor adherence on maternal and family measures. J Child Adolesc Psychopharmacol. 2006;16:286-297.
22. Olson DH. Circumplex Model VII: validation studies and FACES III. Fam Process. 1986;25:337-351.

23. Rothman KJ, Greenland S. Causation and causal inference. In: Rothman KJ, Greenland S, Hartge P, et al, eds. Modern Epidemiology. 2nd ed. New York, NY: Lippincott-Raven; 1998:7-28.

24. Chioqueta AP, Stiles TC. Personality traits and the development of depression, hopelessness and suicide ideation. Pers Individ Dif. 2005:1283-1291.

25. Adams JB, Heath AJ, Young SE, Hewitt JK, Corley RP, Stallings MC. Relationships between personality and preferred substance and motivations for use among adolescent substance abusers. Am J Drug Alcohol Abuse. 2003;29:691-712.

26. Hawton K, Houston K, Haw C, Townsend E, Harriss L. Comorbidity of axis I and axis II disorders in patients who attempted suicide. Am J Psychiatry. 2003;160:1494-1500.

27. Cavanagh JT, Carson AJ, Sharpe M, Lawrie SM. Psychological autopsy studies of suicide: a systematic review. Psychol Med. 2003;33:395-405.

28. Gould MS, King R, Greenwald S, et al. Psychopathology associated with suicidal ideation and attempts among children and adolescents. J Am Acad Child Adolesc Psychiatry. 1998;37:915-923.

29. Vermeiren R, Schwab-Stone M, Ruchkin VV, King RA, Van Heeringen C, Deboutte D. Suicidal behavior and violence in male adolescents: a school-based study. J Am Acad Child Adolesc Psychiatry. 2003;42:41-48.

30. Malone KM, Waternaux C, Haas GL, Cooper TB, Li S, Mann JJ. Cigarette smoking, suicidal behavior, and serotonin function in major psychiatric disorders. Am J Psychiatry. 2003;160:773-779.

31. Light JM, Grube JW, Madden PA, Gover J. Adolescent alcohol use and suicidal ideation: a nonrecursive model. Addict Behav. 2003;28:705-724.

32. Lerman C, Glanz K. Stress, coping and health behavior. In: Glanz K, Lewis FM, Rimer BK, eds. Health Behavior and the Health Education. San Francisco, CA: Jossey-Bass; 1997:113-138.

33. Martin G, Waite S. Parental bonding and vulnerability to adolescent suicide. Acta Psychiatr Scand. 1994;89:246-254.

34. Chao RK. Beyond parental control and authoritarian parenting style: understanding Chinese parenting through the cultural notion of training. Child Dev. 1994;65:1111-1119.

35. Leung K, Lau S, Lam WL. Parenting styles and academic achievement: a cross-cultural study. Merrill-Palmer $Q$. 1998;44:157-172.

36. McKeown RE, Garrison CZ, Cuffe SP, Waller JL, Jackson $\mathrm{KL}$, Addy CL. Incidence and predictors of suicidal behaviors in a longitudinal sample of young adolescents. J Am Acad Child Adolesc Psychiatry. 1998;37:612-619.

37. Martin G, Rozanes P, Pearce C, Allison S. Adolescent suicide, depression and family dysfunction. Acta Psychiatr Scand. 1995;92:336-344.

38. Gau SF, Soong WT. The transition of sleep-wake patterns in early adolescence. Sleep. 2003;26:449-454. 
Copyright of Journal of American College Health is the property of Heldref Publications and its content may not be copied or emailed to multiple sites or posted to a listserv without the copyright holder's express written permission. However, users may print, download, or email articles for individual use. 